\title{
Local ratcheting phenomena in the cyclic behavior of polycrystalline tantalum
}

\author{
Damien Colas, Eric Finot, Sylvain Flouriot, Samuel Forest, Matthieu Mazière, Thomas \\ Paris
}

\begin{abstract}
A direct numerical simulation of the cyclic response of a 250 grain polycrystalline aggregate over more than 1000 cycles is presented, which belongs to the few available simulations including a significant number of cycles. It provides unique results on the evolution of accumulated plastic strain and ratcheting phenomena inside the grains. Even though the average stress strain response is stabilized after 500 cycles, unlimited ratcheting is observed at some locations close to grain boundaries and triple junctions. A clear surface effect of the ratcheting behavior is evidenced based on an appropriate combination of Dirichlet, Neumann and periodic boundary conditions. The magnitude of the ratcheting indicator is found to be significantly higher at the free surface than in the middle section of the aggregate. The studied material is pure tantalum tested at room temperature for both single and polycrystalline samples used for parameter identification of the crystal plasticity model. Special attention is dedicated to the modeling of static strain aging effects observed in this material.
\end{abstract}

\section{Introduction}

The direct simulation of the mechanical behavior of polycrystalline aggregates based on crystal plasticity provides a wealth of information about the development of strain heterogeneity from grain to grain and inside the grains [1]. It can be applied to cyclic loading conditions for the assessment of lifetime criteria from a statistical analysis of simulations

D. Colas, S. Flouriot, T. Paris

CEA Valduc, 21120 Is-sur-Tille, France e-mail: sylvain.flouriot@cea.fr

S. Forest, M. Mazière

MINES ParisTech, PSL Research University, Centre des matériaux, CNRS UMR 7633, BP 8791003 Evry, France e-mail: samuel.forest@mines-paristech.fr

E. Finot

Laboratoire Interdisciplinaire Carnot de Bourgogne, UMR 5209 CNRS, Université de Bourgogne, 9 avenue Alain Savary, BP 17870, 21078 Dijon Cedex, France 
at the grain scale [2]. Local fatigue damage indicators can be post-processed from the field of plastic slip along slip systems in connection with stress and strain values to predict crack initiation or propagation [3,4]. Direct comparison with experiment is possible when considering aggregates constructed from the EBSD analysis of real samples as done recently in [5]. However, due to prohibitive computation times, most simulations are performed for 2 to 10 cycles hoping that it is enough to reach stabilized stress-strain loops at each integration point. Hundred cycles were simulated for copper thin films on a substrate in [6] where plastic shakedown was observed after 50 cycles due to strong cyclic strain hardening of the material and confinement induced by the substrate. In the present work focusing on Low Cycle Fatigue (LCF) loading conditions, 1000 cycles are simulated for a representative polycrystalline aggregate including a free surface under symmetric strain-based overall loading conditions. The simulation provides unique information on the cyclic response of material points inside the grains, close to grain boundaries and to a free surface. Local ratcheting phenomena will be documented in order to decipher the mechanisms of redistribution of stress and strain in polycrystals under cyclic loading. The ultimate goal is to identify microstructure sensitive fatigue crack initiation criteria.

The studied material is pure tantalum which is a refractory material used in several industries such as nuclear, capacitors, lighting, biomedical and chemical processing. Tantalum has been the subject of many experimental and computational studies of crystal plasticity. Large polycrystalline simulation volumes involving massive computations are provided in [7]. Crystal plasticity effects were examined at notches and wedges [8]. However, these studies are dedicated to the monotonic behavior of tantalum in contrast to the aim of the present work. A macroscopic model was recently proposed for the cyclic behavior of tantalum including a specific feature of this material, namely static strain aging effects [9]. The tensile curve and the first cyclic loops exhibit a peak stress followed by softening which is attributed to interaction between dislocations and solute oxygen atoms which are always present in tantalum. Due to the strong rate-dependence effects at room temperature, the softening part of the material response was shown to be associated to limited strain localization in contrast to the usual Lüders phenomenon in steels.

The paper is outlined as follows. The description of the material and experimental analysis of single and polycrystal behavior follows this introduction. The evolution boundary value problem handled in this work is described in detail in Section 3 including the presentation of the crystal plasticity model and the parameter identification procedure. The results are discussed in Section 4 focusing on the description of ratcheting phenomena in the bulk and at a free surface.

\section{Material and experimental results}

The material studied is a commercially pure tantalum $(99.95 \%$ w.) coming from Cabot Performance Materials (USA). The sheet used has been recrystallized during 2 hours between 1000 and $1200^{\circ} \mathrm{C}$ and at $10^{-4}$ to $10^{-5}$ mbar. After recrystallization, the mean grain size is close to $120 \mu \mathrm{m}$ and the initial dislocation density is rather high, between $\rho_{d} \approx 10^{13} \mathrm{~m}^{-2}$ and $\rho_{d} \approx 10^{14} \mathrm{~m}^{-2}[10,11,12]$. In other studies on commercially pure tantalum, the mean 
grain size was about 50 to $70 \mu m[13,14]$ for the same heat treatment conditions. The discrepancy may be due to the different thicknesses of the sheets used in the different studies, leading to different strain levels during sheet production. EBSD maps over large areas (not provided here) show isotropic grain shape without pronounced crystallographic texture due to cross rolling of the sheet. Some curved grain boundaries are observed, probably caused by non-optimal recrystallization conditions.

Interrupted tension-compression cyclic tests have been carried out on thick flat samples to avoid buckling at a strain rate $\dot{\varepsilon}=10^{-3} \mathrm{~s}^{-1}$ and a strain amplitude of $\frac{\Delta \varepsilon}{2}=0.2 \%$. The influence of the strain amplitude on the cyclic behavior has been investigated in a previous paper by the same authors [9]. Strain field measurements and SEM observations have been performed on the sample surface. They will be reported in a future paper for the sake of brevity. The cyclic test has been interrupted after 100, 1000, 2000 and 3000 cycles. Each time, the sample was unloaded from the test machine and placed into a SEM for some secondary, back-scattered and EBSD pictures of each grid. The test machine used was a servo-hydraulic Instron 8800.

The shape of the hysteresis loop does not evolve during the test, with a significant Bauschinger effect, as shown in the right Fig. 1. Isotropic hardening of about $20 \mathrm{MPa}$ reaches a saturation point after about 50 cycles.

Tensile and cyclic tests have also been performed on tantalum single crystals produced by Goodfellow. The corresponding tensile curves are shown in Fig. 1 (left) for tension in the crystallographic directions $<100>$ and $<111>$. They are compared to the tensile curve of the polycrystal material. All curves display an initial peak stress followed by softening and subsequent hardening. This phenomenon has been attributed in [9] to static strain aging due to the presence of solute atoms, especially oxygen. It is well-known that the oxygen content has a dramatic effect on the mechanical behavior of tantalum. A macroscopic constitutive model was proposed in [9] that accounts for strain aging effects in polycrystalline tantalum. Material purity therefore plays a significant role in the understanding of tantalum mechanical response. The chemical composition, determined by IR-combustion or inert gas fusion, is given for all samples in Table 1. The oxygen contents are much below the typical authorized limits of 150ppm (weight) for tantalum applications. This was shown however to be high enough for static strain ageing effects to show up for polycrystals [9]. The lower concentration of oxygen in the single crystals may explain why the peak stresses in Fig. 1a are less pronounced than for the polycrystal. The single crystal tensile tests will be used for the identification of the model parameters in the next section.

\begin{tabular}{|c|c|c|c|}
\hline element & \multicolumn{2}{|c|}{ single crystal } & polycrystal \\
\hline & {$[100]$} & {$[111]$} & \\
$\mathrm{C}$ & 7.4 & $<5$ & 10 \\
$\mathrm{~N}$ & $<5$ & $<5$ & $<10$ \\
$\mathrm{O}$ & $<10$ & 11 & 19 \\
$\mathrm{H}$ & 2.7 & 2.5 & 6 \\
\hline
\end{tabular}

Table 1 Chemical purity in (weight) ppm of the considered tantalum single- and polycrystals 




(a)

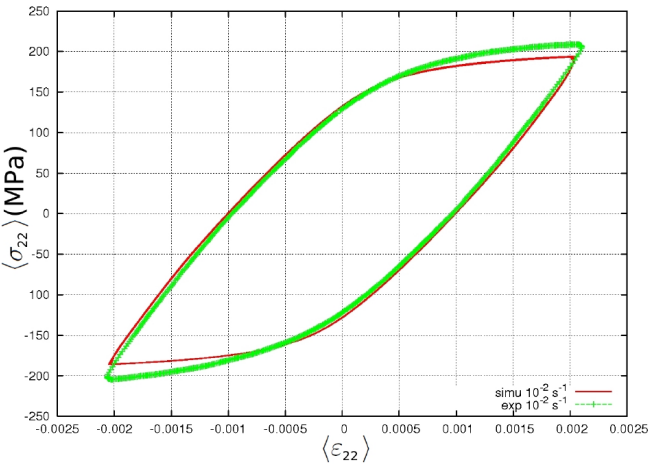

(b)

Fig. 1 (a) Comparison between single crystal and polycrystal behavior under tensile loading at $\dot{\varepsilon}=$ $10^{-2} s^{-1}$; (b) Experimental and simulated stabilized cyclic loops at the same strain rate for the polycrystal (right).

\section{Finite Element simulations}

The finite element simulations of the cyclic behavior of a tantalum polycrystalline aggregate are presented in the following. The description of geometry, constitutive law and loading conditions is followed by results dealing with the evolution of the heterogeneous plastic strain distribution during cycling.

\subsection{Description of semi-periodic polycrystalline aggregates}

The polycrystalline aggregate considered in this work is characterized by two parallel flat surfaces perpendicular to the space direction 3, and four lateral surface displaying periodicity of grain morphology along the directions 1 and 2, as shown in Fig. 2. The throughthickness view of Fig. 2 shows that the grain morphology of the parallel flat surfaces is not periodic. The aggregate of Fig. 2 contains 250 grains with 4 to 5 grains through the thickness in average. The construction of such semi-periodic polycrystalline aggregates starting from a classical Voronoi tessellation follows the strategy proposed by J. Guilhem in the reference [15]. By construction, grain boundaries are flat surfaces ensuring continuity of displacement and reaction forces in finite element simulations.

The three-dimensional finite element mesh is made of quadratic elements (limiting locking effects [16]) with full integration. The mesh of Fig. 2 contains 55089 nodes and 37595 quadratic tetrahedral elements. The number of grains in the polycrystalline volume and the mesh refinement have been chosen so as to ensure sufficient representativity of the polycrystalline response according to previous studies of Representative Volume Element size for f.c.c. crystals $[1,17,18]$. In particular, the thickness was chosen following the works 
$[19,20]$ showing that the strain field at a free surface of a cubic polycrystal is affected mainly by the first three layers of grains below the surface. It should be noted that the previous representativity studies were performed for monotonic loading and that the question of the RVE size should be addressed again in the case of cyclic loading. An attempt to determine the RVE size for cyclically loaded bulk polycrystals can be found in [21] where polycrystalline aggregates made of 100 grains were considered. In the recent work [22] an appropriate statistical approach involving 10 to 250 grains was applied to cyclic crystal plasticity. Finally, it must be noted that only one Finite Element simulation was carried out in this work due to the fact that more than 1000 cycles were simulated leading to computation times not compatible with a statistical analysis for several realizations of the microstructure.



Fig. 2 Synthetic semi-periodic aggregate made of 250 grains: General view (top left), free surface (top right), view through the thickness (bottom). The top and bottom lines in the bottom view are traces of the planes $z=0$ and $z=H$. The numbers (top right picture) denote the finite element nodes studied in section 4.2

\subsection{Boundary conditions}

The two flat surfaces of the polycrystalline aggregate of Fig. 2 are called $Z=0$ and $Z=H$. The surface $Z=0$ is subjected to the Dirichlet boundary condition $u_{3}=0$ where $u_{3}$ is 
the displacement component along the direction 3 . The remaining boundary conditions for this surface $Z=0$ are vanishing traction components along 1 and 2 . All three components of the traction vector on the surface $Z=H$ are set to zero, corresponding to vanishing Neumann boundary conditions. The strain fields on this free surface will be analyzed and compared, in a statistical sense, with the experimental results.

Pairs of homologous points, $\left(\boldsymbol{x}^{-}, \boldsymbol{x}^{+}\right)$, are defined on the four periodic lateral surfaces. The displacements of these points are related by the linear conditions:

$$
u_{i}\left(\boldsymbol{x}^{+}\right)-u_{i}\left(\boldsymbol{x}^{-}\right)=E_{i j}\left(x_{j}^{+}-x_{j}^{-}\right)
$$

thus enforcing periodicity of all three components of the displacement fluctuation with respect to the macroscopic strain, $E_{i j}$. This is done by elimination of redundant degrees of freedom according to the usual multi-point constraint in a finite element code. The vector $x_{i}^{+}-x_{i}^{-}$is either parallel to the axis 1 or to the axis 2, corresponding to the two directions of periodicity, and its magnitude is equal to the width of the aggregate. Periodic boundary conditions are used for the lateral faces because they are known to limit boundary layer effects in the simulation of material volume elements in contrast to homogeneous Dirichlet or Neumann conditions, see [23, 24, 25]. They lead to smaller Representative Volume Element sizes in general [23]. Due to the periodic boundary conditions in the 1-2 directions it is also necessary to fix one node $\left(u_{1}=u_{2}=0\right)$ to set the rigid body translation which is indeterminate in periodic computations.

The average stress and total strain components over the whole volume element, $V$, are computed as

$$
\Sigma_{i j}=<\sigma_{i j}>=\frac{1}{V} \int_{V} \sigma_{i j} d V, \quad E_{i j}=<\varepsilon_{i j}>=\frac{1}{V} \int_{V} \varepsilon_{i j} d V
$$

The macroscopic total strain component $E_{22}= \pm 0.2 \%$ is imposed at the strain rate $10^{-2} \mathrm{~s}^{-1}$ (LCF). The components $E_{11}$ and $E_{12}$ are treated as additional degrees of freedom in the finite element method for periodic homogenization, see [26]. They are determined at each increment so as to ensure that the conjugate average stress components vanish: $\Sigma_{11}=\Sigma_{12}=$ 0 . As a consequence of the boundary conditions, it can be shown that the average stress components $\Sigma_{33}=\Sigma_{13}=\Sigma_{23}$ also vanish. The loading conditions therefore correspond to a simple tension-compression test with one free surface, four periodic surfaces and one flat surface.

The results presented in this work correspond to the simulation of more that $1000 \mathrm{cy}-$ cles on the previously described polycrystalline aggregate. They represent 11 months of computation time on a 12 core Intel Xeon $3 \mathrm{GHz}$ processor with 25 Go RAM. 1250 time steps were saved for 70 variables (stress, strain and plastic strain tensor components, slip amounts, accumulated slip amounts, kinematic hardening variables, accumulated plastic strain...) at each integration point, which amounts to 250Go disk space. 


\subsection{Crystal plasticity model}

The constitutive equations of the crystal plasticity model used for tantalum are now given. The Cailletaud crystal plasticity model [27] is extended here to include static strain aging effects as displayed by tantalum crystals. The strain tensor is written as the sum of the elastic and plastic contributions:

$$
\varepsilon_{i j}=\varepsilon_{i j}^{e}+\varepsilon_{i j}^{p}
$$

The local elastic behavior exhibits cubic symmetry, with an elasticity tensor characterized by the three independent elastic moduli $C_{11}=267 \mathrm{MPa}, C_{12}=159 \mathrm{MPa}$ and $C_{44}=83 \mathrm{MPa}$, after [28]. The plastic strain tensor results from plastic slip processes with respect to all slip systems. In the present work, 12 slip systems are considered on $\{110\}$ slip planes with slip directions $<111>$ corresponding to the b.c.c. structure. This choice represents a simplification since more slip planes are known to be available in b.c.c. crystals [29]. Motivations for the present choice can be found in [30] where the selection of $\{110\}$ vs. $\{112\}$ planes is discussed. The limitation of the number of slip systems is also due to the necessary reduction of the computational cost of the presented simulations for the simulation of high number of cycles. The viscoplastic strain rate tensor is written as:

$$
\varepsilon_{i j}^{p}=\sum_{s} \dot{\gamma}^{s} m_{i j}^{s}, \quad \text { with } \quad m_{i j}^{s}=\frac{1}{2}\left(\ell_{i}^{s} n_{j}^{s}+n_{i}^{s} \ell_{j}^{s}\right)
$$

where $n_{i}^{s}$ is the normal vector to the slip plane and $\ell_{i}^{s}$ the slip direction, for the slip system number $s$. The slip rate for each slip system is evaluated using the following viscoplastic law:

$$
\dot{\gamma}^{s}=\dot{v}_{0} \sinh \left(\frac{\left|\tau^{s}-x^{s}\right|-r^{s}-r_{a}}{\sigma_{0}}\right) \operatorname{sign}\left(\tau^{s}-x^{s}\right), \quad \dot{v}^{s}=\left|\dot{\gamma}^{s}\right|
$$

where $\tau^{s}=\sigma_{i j} m_{i j}^{s}$ is the resolved shear stress for slip system $s$ and $v^{s}$ is the cumulative slip variable. The slip rate is driven here by the resolved shear stress only and non-Schmid effects, sometimes observed in b.c.c. crystals, are not introduced here for simplicity [31, 32], as done in [30]. The isotropic hardening follows a non linear evolution law:

$$
r^{s}=r_{0}+Q \sum_{r} h^{s r}\left(1-\exp \left(-b v^{r}\right)\right)
$$

where $r_{0}$ is the initial critical resolved shear stress, $Q$ and $b$ are responsible for hardening; $h^{s r}$ is the interaction matrix which characterizes both self-hardening and cross-hardening between the different slip systems.

The kinematic hardening term is the main ingredient for the description of internal stresses building up inside the grains, for instance due to dislocation pile-ups or/and dislocation structure formation [33]. It is decomposed into two contributions for each slip system $s$ :

$$
x^{s}=x_{1}^{s}+x_{2}^{s}
$$

The evolution equation of each component is: 


$$
\dot{x}_{i}^{s}=c_{i} \dot{\gamma}^{s}-d_{i} x_{i}^{s} \dot{v}^{s}, \quad i \in\{1,2\}
$$

where $c_{i}$ and $d_{i}$ are kinematic hardening material parameters. The reason for introducing two components $x_{1}^{s}$ and $x_{2}^{s}$ is the need for a better description of internal stress effects at very low strain levels close or below the apparent yield stress, and for larger strain levels, as it will be seen in the identification of material parameters.

The previous crystal plasticity model is now enhanced by the addition of a resistance term associated with static strain aging, namely the component $r_{a}$ in Eq. (5). This additional hardening term is related to a new internal variable, $t_{a}$, called the aging time, as proposed by $[34,35,36,37,38]$ for polycrystals. The relation between $r_{a}$ and the aging time variable, and its evolution equation are:

$$
r_{a}=P_{1}\left(1-\exp \left(-P_{2} t_{a}^{\beta}\right)\right), \quad \text { with } \quad \dot{t}_{a}=1-\frac{t_{a} \dot{\varepsilon}_{e q}^{p}}{\omega}
$$

The variable $r_{a}$ increases with the aging time $t_{a}$, the state of totally pinned dislocations being given by $r_{a}=P_{1}$. The unpinned state corresponds to $r_{a}=0$. The maximal additional stress required to unpin dislocations from solute atoms is driven by the parameter $P_{1}$. It can be related to the interaction force between immobilized dislocations and solute atoms. The parameters $P_{2}$ and $\beta$ control the kinetics of the pinning/unpinning processes. Another parameter $\omega$ appears in the evolution law of the aging time and is related to the incremental slip resulting from the jump of unpinned dislocations. This parameter is kept constant in the present work. The evolution equation $(9)_{2}$ involves the equivalent plastic strain rate defined as:

$$
\dot{\varepsilon}_{e q}^{p}=\sqrt{\frac{2}{3} \dot{\varepsilon}_{i j}^{p} \dot{\varepsilon}_{i j}^{p}}
$$

The initial condition for the differential equation driving $t_{a}$ is $t_{a}(t=0)=t_{a 0}$. For the sake of simplicity, a practical formulation has been adopted, with a single aging time $t_{a}$ for all slip systems, as done in [37]. As a consequence, the aging shear stress $r_{a}$ is the same for all slip systems and depends on the material parameters $P_{1}, P_{2}, \beta$. The evolution of $t_{a}$ requires the identification of the material parameter $\omega$. In the case of static strain aging, $t_{a}$ has a large initial value $t_{a 0}$ that represents the initial "pinned state". In this work this initial value is assumed to be equivalent to fully pinned, according to the aging experiments presented in a previous study [9]. In the latter reference, oxygen was considered as the solute atom responsible for aging effects in tantalum.

The aging single crystal model has been implemented in the implicit finite element code Zset [39], following the numerical procedure presented in [26, 37].

Finally, the equivalent plastic strain is defined as the Euclidean norm of the plastic strain tensor and will be used for the post-processing of the finite element results:

$$
\varepsilon_{e q}^{p}=\sqrt{\frac{2}{3} \varepsilon_{i j}^{p} \varepsilon_{i j}^{p}}
$$




\subsection{Identification of material parameters}

The previous crystal plasticity model including strain aging effects involves 15 parameters that must be identified. Two groups of parameters are distinguished, namely those related to the standard crystal plasticity hardening and those concerning the strain aging part. The parameter identification procedure takes the mechanical tests for both single crystals and polycrystals into account. The monotonic and cyclic tests on tantalum single crystals were presented in section 2. The three monotonic tensile tests (strain rates: $10^{-4} \mathrm{~s}^{-1}, 10^{-3} \mathrm{~s}^{-1}$, $10^{-2} \mathrm{~s}^{-1}$ ) and the three stabilized cyclic loops (strain amplitudes: $\pm 0.0018, \pm 0.0025, \pm 0.005$ at the strain rate at $10^{-3} \mathrm{~s}^{-1}$ ) for polycrystalline tantalum presented in the reference [9] are used for the identification of the single crystal behavior. For that purpose, a periodic 50 grain polycrystalline aggregate was used to perform fast running finite element simulations using the single crystal model at the grain level. The optimization procedure using the Levenberg-Marcquardt algorithm involves evaluation of the polycrystalline aggregate response and the comparison with the experimental results. The material parameters obtained using this procedure provide single crystal responses in agreement with the single crystal experiments. The polycrystalline response was favored in the identification because it concerns the polycrystalline material whose cyclic behavior is examined in the present paper. Once satisfactory parameters are found based on the small 50-grain aggregates, simulations are performed on a 250-grain aggregate to test the representativity of the results. Differences below $20 \mathrm{MPa}$ were observed leading to a final tuning of the parameters. Two kinematic hardening components were identified, $x_{1}$ and $x_{2}$ with parameters $c_{1}, c_{2}, d_{1}, d_{2}$, for a better description of the hysteresis loops. Due to the very small cyclic hardening for strain amplitudes close to $\pm 0.2-0.25 \%$, the isotropic hardening parameter, $Q$, turns out to be almost negligible. All the components of the interaction matrix $h^{r s}$ in Eq. (6) were set to 1 following Taylor's assumption, as done in $[30,40,8]$ for tantalum in the absence of sufficient experimental testing for the identification of interactions between slip systems. It can be noted that the initial critical resolved shear stress $r_{0}$ was set to zero since it turns out that the apparent yield stress is mostly accounted for by the strain aging term $r_{a}$. Also, the kinematic hardening component $x_{1}$ with a very high hardening modulus $c_{1}$ provides a quasi-linear apparent initial part in the tensile curves. It is well-known that slip activity in grains starts very early even though the polycrystal response is apparently quasi-linear, with important implications for HCF.

We now come to the second group of parameters characterizing the strain aging part of the model. The initial value of the aging time $t_{a 0}$ is set so as to ensure a sufficiently large initial pinning state, in the present case $t_{a 0}=5 \cdot 10^{4} \mathrm{~s}$, following [41]. At the beginning of test simulation, $r_{a}$ is therefore close to $P_{1}$. As soon as plastic strain occurs, $t_{a}$ and then $r_{a}\left(t_{a}\right)$ start decreasing to an asymptotic value $r_{a}\left(p, t_{w}=\omega / \dot{\varepsilon}_{e q}^{p}\right)$ due to Eq. 9 which leads to a stress drop. The parameter $\omega=4 \cdot 10^{-4}$ is set according to the literature $[41,42,43]$ for steels but this value was found to be satisfactory for the macroscopic strain aging model proposed in [9] for tantalum. The parameter $P_{1}=70 \mathrm{MPa}$ controls the aging contribution to the yield stress and therefore the maximum stress drop amplitude, whereas $P_{2}=0.005 s^{-\beta}$ controls the final value of $r_{a}\left(p, t_{a}\right)$ and is set in order to ensure a small residual contribution $r_{a}$ when the unpinning process occurs. The found material parameters are given in table 2. 
The curves for comparison between aggregate simulations and experimental results are not provided here since the quality of identification is close to the one obtained in [9] using a macroscopic model. One of them is provided in Fig. 1. In particular the material parameters of the single crystal model lead to satisfactory description of the peak stress and stress decrease observed in the tensile curves for single crystals in Fig. 1.

\begin{tabular}{|c|c||c|c||c|c|}
\hline$\dot{v}_{0}$ & $5 \cdot 10^{-5} s^{-1}$ & $c_{1}$ & $360 \cdot 10^{3} \mathrm{MPa}$ & $t_{a 0}$ & $5 \cdot 10^{4} s$ \\
$\sigma_{0}$ & $5 \mathrm{MPa}$ & $D_{1}$ & 8000 & $P_{1}$ & $40 \mathrm{MPa}$ \\
$r_{0}$ & $0 \mathrm{MPa}$ & $C_{2}$ & $250 \mathrm{MPa}$ & $P_{2}$ & $0.005 s^{-\beta}$ \\
$Q$ & $1 \mathrm{MPa}$ & $D_{2}$ & 1.5 & $\omega$ & $4 \cdot 10^{-4}$ \\
$b$ & 1.5 & $h^{r s}$ & 1 & $\beta$ & 0.66 \\
\hline
\end{tabular}

Table 2 Aging single crystal model parameters identified for tantalum at room temperature.

\subsection{Simulation of the mechanical fields at the free surface}

The overall stress-strain loop, $\left\langle\sigma_{22}>-<\varepsilon_{22}>\right.$, of the studied cyclic test on the considered polycrystalline aggregate is given in Fig. 1. Under the symmetric strain based loading conditions, a stabilized loop is obtained at the macroscale. The stress and strain amplitudes are clearly visible. The loops after 66, 466 and 1066 cycles (not presented here) are almost identical showing that no macroscopic ratcheting takes place for the considered loading. The first loop is strongly different due to the initial peak stress associated with static strain aging.

The evolution of the several mechanical variables on the free surface of the polycrystal is now discussed with respect to the number of cycles. All surface fields are provided for a vanishing value of the mean axial stress $\Sigma_{22}=\left\langle\sigma_{22}\right\rangle=0 \mathrm{MPa}$ (unloaded state) in accordance with the experimental procedure.

\section{Surface strain field}

The fields of the total strain components $\varepsilon_{11}, \varepsilon_{22}$ and $\varepsilon_{12}$ at the free surface of the polycrystalline aggregate are shown in Fig. 3. Three snapshots are presented at the cycle numbers 66, 666 and 1066 for each component. The residual strain field is strongly heterogeneous with axial strain values from -0.003 to more than 0.005 including local hot spots where these values are significantly higher than the loading amplitude \pm 0.002 . This strain heterogeneity is found to increase significantly between $N_{\text {cycle }}=66$ and $N_{\text {cycle }}=666$, with certain grains concentrating more deformation, especially at some grain boundaries. The localization remains the same after 400 additional cycles but the contrast intensifies between tensile and compression zones. The heterogeneity culminates for the shear component. It is expected that higher strain levels would be reached using a finer mesh. Moreover, some ar- 
(a)

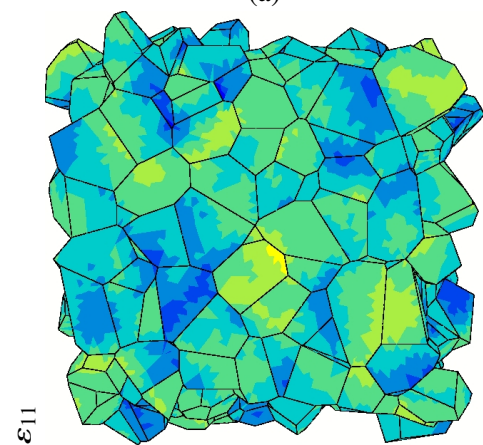

(b)



(c)

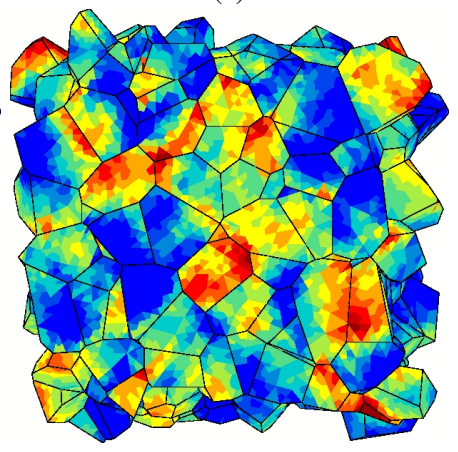

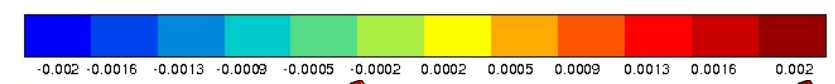
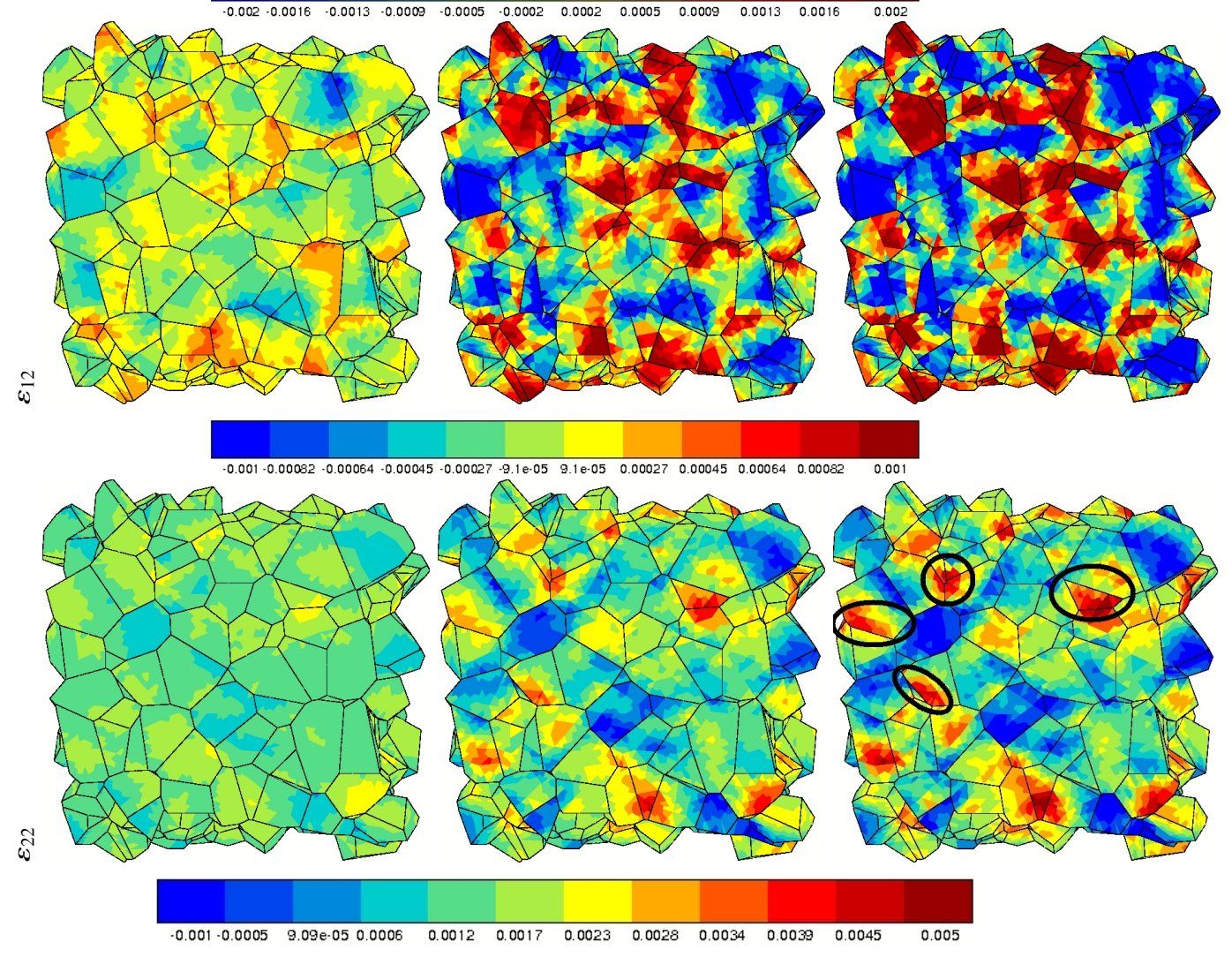

Fig. 3 Evolution of the total strain components at Gauss points at the free surface for (a) cycle 66, (b) cycle 666 and (c) cycle 1066 during a fatigue test simulation at $\pm 0.2 \%$ and $10^{-2} \mathrm{~s}^{-1}$. The loading direction 2 is vertical. The mean stress value is zero (unloaded state). 
eas close to grain boundaries and triple junctions display discontinuous strain values due to crystallographic orientation jump at the interface and resulting strain incompatibility (black circles on the $\varepsilon_{22}$ component). This strain localization close to grain boundaries is shown to increase with the number of cycles, see for instance the circled regions of Fig. 3. Significant roughening of the surface is predicted in agreement with previous cyclic behavior simulations of copper in [6].

The surface deformation can also be analyzed in terms of plastic deformation. The plastic slip activity is found to increase drastically over the more than thousand cycles. Significant evolution in plastic slip activity is observed between the cycles 666 and 1066 . The effect is stronger than for the strain fields of Fig. 3. When the number of cycles increases, the slip increments decrease but remain positive and strongly heterogeneous. These features of plastic slip accumulation are discussed in the next section.

\section{Discussion}

The salient features of the computational results are now discussed, namely the difference between bulk and free surface plastic activity, local ratcheting phenomena at the free surface, and the implications for the initiation of fatigue cracks.

\subsection{Evidence of a free surface effect}

The difference between bulk and surface straining responses is highlighted in Fig. 4 where maps of total strain component $\varepsilon_{22}$ are given at the free surface and in the mid-section for three numbers of cycles. Strong strain heterogeneity in the form of strain bands inclined at about $45^{\circ}$ is observed in the bulk. These bands correspond to the usual observations in the analysis of polycrystalline aggregates, both from the computational point of view [18] and from the experimental one [44]. The strain heterogeneity is much stronger at the free surface and accumulate with increasing number of cycles. The same bands can be observed on the free surface and in a parallel plane in the bulk (first and second rows in Fig. 4), with the difference that the strain heterogeneity inside these bands becomes larger at the free surface. In particular, heterogeneity at grain boundaries is more pronounced than in the bulk. This difference of bulk and free behavior will be confirmed quantitatively in the two next subsections.

The question arises about the impact of static strain aging on the evidenced difference in the bulk/free surface behavior. For that purpose, a computation of the same aggregate was performed under the same conditions but without the aging term $\left(t_{a}=0\right)$. It shows that aging affects the responses up to 100 cycles. Later on, the strain fields are almost identical. This provides evidence that the static strain aging does not affect the long-term material response. However, a more thorough analysis is required to check the evolution of field of ageing time variable $t_{a}$ during cycling. A tensile test could be simulated and performed 
(a)
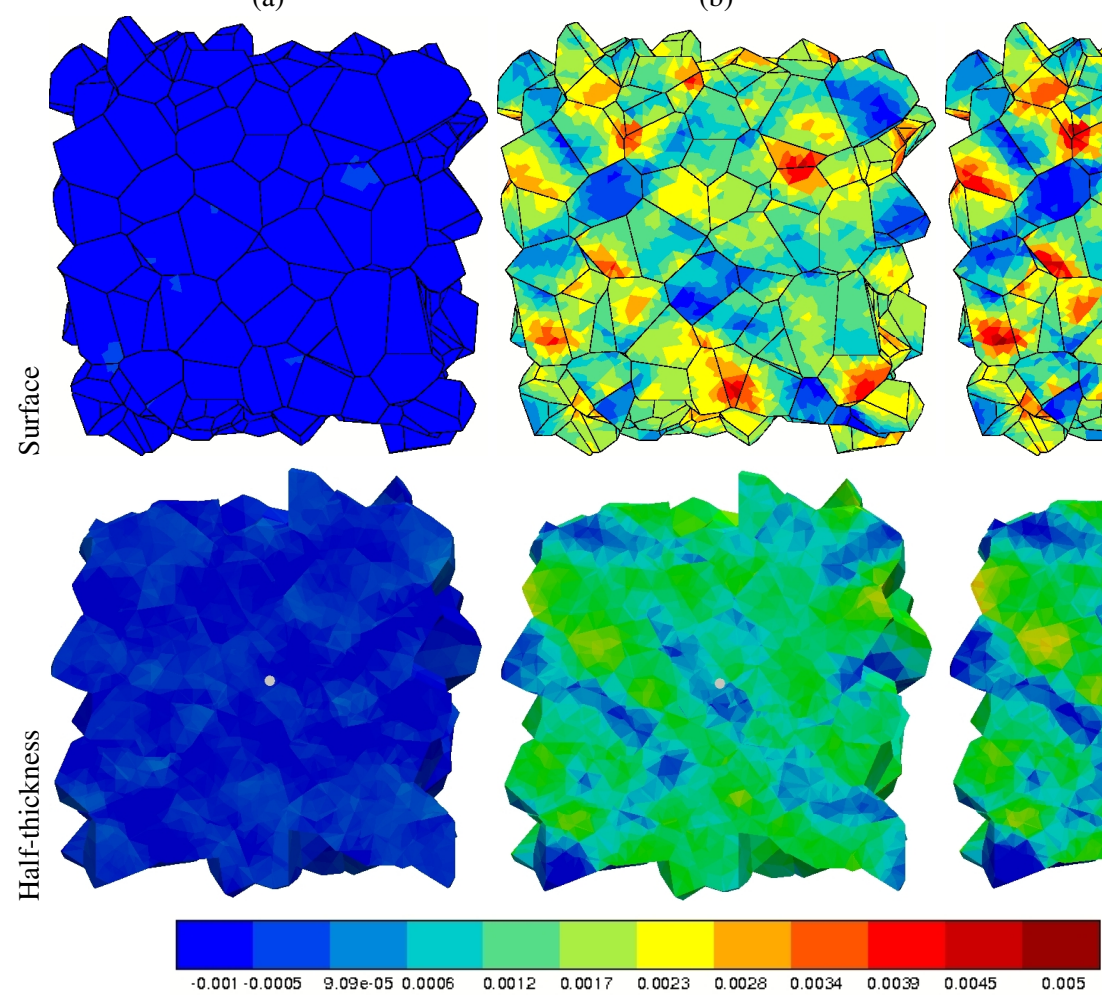

Fig. 4 Strain field $\varepsilon_{22}$ evolution at (a) cycle 66, (b) cycle 666 et (c) cycle 1066 at the free surface (top row) and half-thickness (bottom row). The loading direction, corresponding to axis 2, is vertical. The mean stress value is zero (unloaded state).

experimentally at the end of the cyclic to test the existence or not of a peak stress in the tensile curve.

\subsection{Local ratcheting behavior}

Accumulation of plastic strain is of the utmost importance for crack initiation. It is proved in this subsection that ratcheting events are significantly more pronounced at the free surface than in the bulk.

\section{Local study at several finite element nodes}

From the previous observation of the free surface, 6 nodes have been selected in order to document the local cyclic response of material points, see Fig. 2. Among the 6 nodes, 3 are 

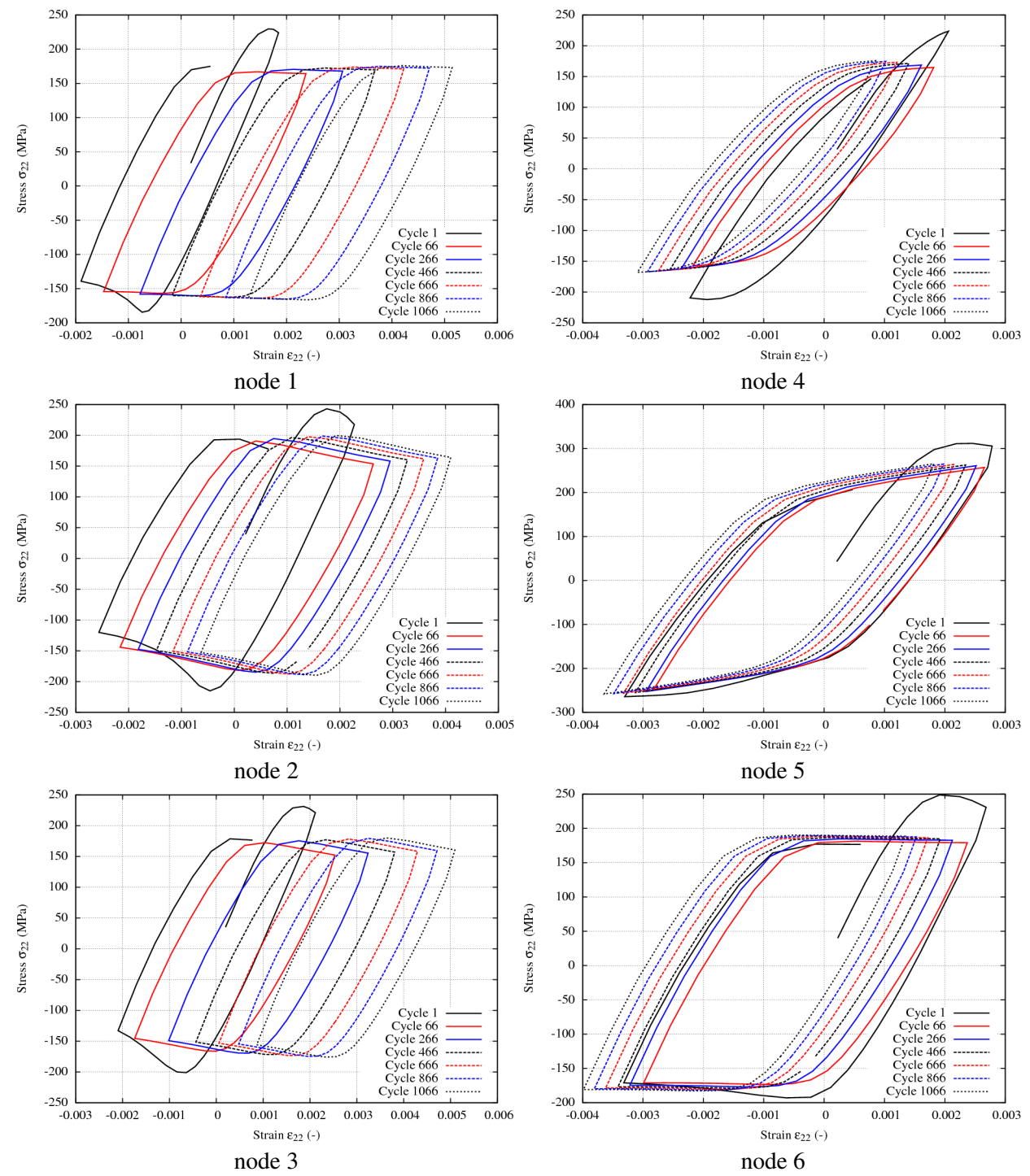

Fig. 5 Local curves $\sigma_{22}-\varepsilon_{22}$ for several cycles at 6 different finite element nodes (see Fig. 2 for their location). 



Fig. 6 Local curves $\sigma_{11}-\varepsilon_{11}$ for several cycles at 6 different finite element nodes (see Fig. 2 for their location).

located in plastically active zones (nodes 1,2 and 3), whereas the other three are situated in less active areas (nodes 4, 5 and 6). This can be inferred from the cyclic stress-strain $\sigma_{22}$ vs. $\varepsilon_{22}$ loops of Fig. 5. All nodes display largely open hysteresis loops revealing significant plastic deformation. The first loop is characterized in each case by the peak stress associated with static strain aging. The amplitude of the stress drop is close to $40 \mathrm{MPa}$, in agreement with the $P_{1}$ parameter identified in Tab. 2 . The first unloading occurs before the end of the unpinning, so that stress softening continues during the first compression phase. This phenomenon has been experimentally observed in [9]. Then the loops become symmetric 
with respect to the stress, due to the relaxation of the mean stress. In contrast, the minimal and maximal strain levels are not symmetric. The loops are not saturated after 1000 cycles and strain accumulates either in tension or compression, thus revealing local ratcheting phenomena. Significant tensile rachteting is found for the nodes 1,2 and 3, meaning that the mean strain is positive and increases monotonically, whereas more limited ratcheting in compression is observed for the nodes 4, 5 and 6 . The local stress-strain loops strongly differ from the macroscopic loop of Fig. 1 which is fully symmetric. Local stress levels $\sigma_{22}$ depend on the crystallographic orientation of each grain and on the interaction with neighboring grains.

The ratcheting phenomenon (also called cyclic creep, although the rate-dependent behavior is not responsible for it) occurs when the plastic strain increment is not fully reversed for a cyclic loading [45]. It happens for instance for non-vanishing mean stress loading: a positive (resp. negative) ratcheting occurs for a positive (resp. negative) mean stress [46, 47, 48]. Several models have been proposed in order to account for ratcheting. They are based on the introduction of one (or more) linear or non-linear kinematic hardening $[46,49,50,51]$. The situation is quite different here since the macroscopic loading is symmetric in strain and stress, at least after a few cycles, see Fig. 1, but, due to strain heterogeneity induced by crystal plasticity inside the grains, the local loops are initially non symmetric and promote ratcheting. However, the mean stress is found to relax to zero in all the plotted loops after less than 100 cycles. The origin of the continuing ratcheting is the multi-axial loading experienced by the material points. Looking at the $\sigma_{11}-\varepsilon_{11}$ loops shown in Fig. 6, the transverse stress component is found to be significant for the six selected nodes. Transverse strain ratcheting is observed for the nodes 1,2 and 3. Similar observation was made for the shear component so that it can be concluded that the material points experience multi-axial ratcheting, responsible for plastic strain accumulation. This plastic strain accumulation is anticipated to ultimately lead to fatigue crack initiation.

\section{Ratcheting indicator}

The progressive accumulation of plastic strain at the surface during strain controlled cycling is regarded in this work as the relevant physical mechanism for fatigue crack initiation in the grains of a polycrystal. It has been reported that it can lead to crack nucleation and finally to the global failure of a structure, see $[45,52,51,48,53,54]$, in accordance with the experimental observations of the present work [55]. A measure of local ratcheting has been proposed in the reference [48] for the analysis of plasticity induced by fretting contact. For that purpose, the local plastic deformation increment $\left(\Delta \varepsilon_{i j}^{p}\right)_{\text {ratch }}$ is defined at each material point for each component of the plastic strain tensor as

$$
\left(\Delta \varepsilon_{i j}^{p}\right)_{\text {ratch }}=\left.\varepsilon_{i j}^{p}\right|_{\text {end of the cycle }}-\left.\varepsilon_{i j}^{p}\right|_{\text {start of the cycle }}
$$

The effective plastic strain increment is then computed as :

$$
\Delta \varepsilon_{\text {ratch }, \text { eff }}^{p}=\sqrt{\frac{2}{3}\left(\Delta \varepsilon_{i j}^{p}\right)_{\text {ratch }}\left(\Delta \varepsilon_{i j}^{p}\right)_{\text {ratch }}}
$$


(a)


Fig. 7 Field of the effective plastic strain increment $\Delta \varepsilon_{\text {ratch,eff }}^{p}$ : (a) at the free surface, (b) at halfthickness, for the cycles 66, 666 and 1066. 
where repeated indices are summed up. It can be computed for each individual cycle. It is regarded as an indicator of local ratcheting since departure of $\Delta \varepsilon_{\text {ratch, eff }}^{p}$ from zero is a signature of accumulation of plastic stain.

The field of effective plastic strain increment is plotted in Fig. 7 for three different cycle numbers. The fields after 666 and 1066 cycles are quite similar indicating persistent ratcheting at some locations of the grains, especially at the free surface. The difference in ratcheting behavior at the free surface and in the bulk is apparent in Fig. 7. The magnitude of ratcheting is found to be significantly smaller in the bulk than at the free surface. The ratcheting rate is significant even after 66 cycles and high values are reached in the zones of high plastic activity. It is remarkable that constant values of ratcheting are reached at some locations, as can be seen from the comparison of the maps at 666 and 1066 cycles. Fatigue crack initiation may be anticipated at such places after a sufficient number of cycles.

The evolution of $\Delta \varepsilon_{\text {ratch,eff }}^{p}$ for the six selected nodes during the fatigue test simulation was analyzed. Two main trends are observed again: the nodes 1,2,3 undergo a strong ratcheting effect, a value of almost $6 \times 10^{-6}$ per cycle which is almost constant after 600 cycles; the nodes $4,5,6$ also exhibit ratcheting but at a lower level of $2 \times 10^{-6}$ per cycle. The ratcheting level is very high for the first cycles and decreases to reach almost constant values after 400 cycles.

The highest ratcheting values are observed mainly close to grain boundaries and at triple junctions, as shown by the fields of effective plastic increment maps of Fig. 7 where several yellow zones are observed at such locations.

\section{Conclusions}

The main results of the present work are the following:

1. A single crystal plasticity model incorporating strain aging effects has been proposed for tantalum at room temperature. It has been identified from tensile and cyclic experimental tests for single and polycrystals. The model accounts for the initial peak stress and following stress softening during the first cycles and for the stabilized hysteresis loops.

2. The three-dimensional Direct Numerical Simulation of polycrystalline aggregate under cyclic loading for more than 1000 cycles belongs to the very few similar simulations available in the literature, bringing new insights especially regarding ratcheting phenomena. Due to the complexity of the material law including aging effects, the computation time for this 250 grain sample was more than 11 months on a single machine.

3. The results reveal that, even though the macroscopic stress-strain loop is stabilized after 500 cycles, local ratcheting behaviors are observed inside the grains, especially close to grain boundaries and triple junctions.

4. The number of grains through the thickness of the sample was large enough to evidence clear free surface effects. For that purpose, suitable combinations of periodic, Dirichlet and Neumann boundary conditions were adopted. Ratcheting plastic increments were found to be significantly larger at the free surface than in the middle section. This essential feature of fatigue behavior is reported here for the first time based on polycrystalline 
aggregate simulations. A definitive conclusion requires a more complete statistical analysis based on several similar simulations or larger volume sizes.

5. The strain aging effect was found to affect only the first cyclic loops and unpinned behavior was observed in the long term material response.

The found values of the ratcheting indicator can be used to identify a fatigue crack initiation criterion from available experimental observations of the free surface of tantalum samples, at least for the uniaxial loading considered in the performed experiments. This will be reported in a forthcoming work where the simulations results will also be compared in detail to experimental observations of tantalum grains deforming on a free surface. The conclusions of this work are limited by the fact that only one single sample was considered so that additional simulations are necessary, possibly incorporating parallel computing and reduced order modeling, to consolidate the findings. The presented calculation can serve as a validation for accelerating methods for the simulation of high cycle numbers like the wavelet approach in [56]. The present contribution devoted to cyclic loading is a useful complement to recent works mainly dealing with the monotonic behavior of tantalum [57]. The developed concepts of combined aging and cyclic plasticity are applicable to other class of materials such as titanium alloys [37].

\section{References}

1. F. Barbe, S. Forest, and G. Cailletaud. Intergranular and intragranular behavior of polycrystalline aggregates. Part 2: Results. International Journal of Plasticity, 17:537-563, 2001.

2. A. Hor, N. Saintier, C. Robert, T. Palin-Luc, and F. Morel. Statistical assessment of multiaxial hcf criteria at the grain scale. International Journal of Fatigue, 67:151 - 158, 2014.

3. D.L. McDowell. Simulation-based strategies for microstructure-sensitive fatigue modeling. Materials Science and Engineering, A468:4-14, 2007.

4. H. Proudhon, J. Li, F. Wang, A. Roos, V. Chiaruttini, and S. Forest. 3D simulation of short fatigue crack propagation by finite element crystal plasticity and remeshing. International Journal of Fatigue, 82:238-246, 2016.

5. S. Basseville, G. Cailletaud, T. Ghidossi, Y. Guilhem, E. Lacoste, H. Proudhon, L. Signor, and P. Villechaise. Numerical analysis on the local mechanical fields in polycrystalline $316 \ln$ stainless steel under cyclic fatigue loading: Comparison with experimental results. Materials Science and Engineering: A, 696:122 - 136, 2017.

6. F. Šiška, S. Forest, P. Gumbsch, and D. Weygand. Finite element simulations of the cyclic elastoplastic behavior of copper thin films. Modelling and Simulation in Materials Science and Engineering, 15:S217-S238, 2007.

7. H. Lim, H. J. Bong, S. R. Chen, T. M. Rodgers, C. C. Battaile, and J. M. D. Lane. Direct numerical simulations in solid mechanics for quantifying the macroscale effects of microstructure and material model-form error. JOM, 68:1427-1445, 2016.

8. H Lim, R Dingreville, L A Deibler, T E Buchheit, and C C Battaile. Investigation of grain-scale microstructural variability in tantalum using crystal plasticity-finite element simulations. Computational Materials Science, 117:437-444, 2016.

9. D. Colas, E. Finot, S. Forest, S. Flouriot, M. Mazière, and T. Paris. Investigation and modelling of the anomalous yield point phenomenon in pure tantalum. Materials Science and Engineering, A615:283295, 2014.

10. W. Wasserbach. Work-hardening and dislocation behaviour of tantalum and tantalum alloys. The minerals, Metals and Materials Society, 1996. 
11. M.N. Norlain. Comportement mécanique du tantale, texture et recristallisation. $\mathrm{PhD}$ thesis, Travaux non-publiés, 1999.

12. C. Kerisit. Analyse de recristallisation statique du tantale déformé à froid pour une modélisation en champ moyen. PhD thesis, Ecole Nationale Supérieure de Mines de Paris, 2012.

13. F. Buy. Etude expérimentale et modélisation du comportement plastique du tantale. Prise en compte de la vitesse de déformation et de l'histoire de chargement. $\mathrm{PhD}$ thesis, Université de Metz, 1996.

14. S. Frénois. Modélisation polycristalline du comportement mécanique du tantale. Application à la mise en forme par hydroformage. PhD thesis, Centrale Paris, 2001.

15. Y. Guilhem, S. Basseville, F. Curtit, J.M. Stephan, and G. Cailletaud. Numerical investigations of the free surface effect in three-dimensional polycrystalline aggregates. Computational Materials Science, 70:150-162, 2013.

16. J. Cheng, A. Shahba, and S. Ghosh. Stabilized tetrahedral elements for crystal plasticity finite element analysis overcoming volumetric locking. Computational Mechanics, 57:733-753, 2016.

17. F. El Houdaigui, S. Forest, A.-F. Gourgues, and D. Jeulin. On the size of the representative volume element for isotropic elastic polycrystalline copper. In Q.S. Zheng Y. Bai and Y.G. Wei, editors, IUTAM Symposium on Mechanical Behavior and Micro-Mechanics of Nanostructured Materials, pages 171180, Beijing, China, 2007. Springer.

18. F. Barbe, R. Quey, A. Musienko, and G. Cailletaud. Three-dimensional characterization of strain localization bands in high-resolution elastoplastic polycrystals. Mechanics Research Communications, 36:762-768, 2009

19. A. Zeghadi, F. Nguyen, S. Forest, A.-F. Gourgues, and O. Bouaziz. Ensemble averaging stress-strain fields in polycrystalline aggregates with a constrained surface microstructure-Part 1: Anisotropic elastic behaviour. Philosophical Magazine, 87:1401-1424, 2007.

20. A. Zeghadi, S. Forest, A.-F. Gourgues, and O. Bouaziz. Ensemble averaging stress-strain fields in polycrystalline aggregates with a constrained surface microstructure-Part 2: Crystal plasticity. Philosophical Magazine, 87:1425-1446, 2007.

21. K. S. Zhang, J. W. Ju, Y. L. Bai, and W. Brocks. Micromechanics based fatigue life prediction of a polycrystalline metal applying crystal plasticity. Mechanics of Materials, 85:16-37, 2015

22. M. Bouchedjra, T. Kanit, C. Boulemia, A. Amrouche, and M. El Amine Belouchrani. Determination of the RVE size for polycrystal metals to predict monotonic and cyclic elastoplastic behavior: Statistical and numerical approach with new criteria. European Journal of Mechanics - A/Solids, 72:1 - 15, 2018.

23. T. Kanit, S. Forest, I. Galliet, V. Mounoury, and D. Jeulin. Determination of the size of the representative volume element for random composites : statistical and numerical approach. International Journal of Solids and Structures, 40:3647-3679, 2003.

24. C. Gerard. Mesures de champs et identification de modèles de plasticité cristalline. PhD thesis, Université Paris 13, 2008.

25. Y. Guilhem, S. Basseville, F. Curtit, J.M. Stephan, and G. Cailletaud. Investigations of the effect of grain clusters on fatigue crack initiation in plycrystals. International Journal of Fatigue, 32:1748$1763,2010$.

26. J. Besson, G. Cailletaud, J.-L. Chaboche, S. Forest, and M. Blétry. Non-Linear Mechanics of Materials. Solid Mechanics and Its Applications 167. Springer-Verlag Berlin Heidelberg, 2009.

27. L. Méric and G. Cailletaud. Single crystal modeling for structural calculations. Part. 2 : finite element implementation. Journal of Engineering Materials and Technology, 113:171-182, 1991.

28. V. Eyraud, M. H. Nadal, and C. Gondard. Texture measurement of shaped material by impulse acoustic microscopy. Ultrasonics, 38:438-442, 2000.

29. T. Hoc, J. Crépin, L. Gélébart, and A. Zaoui. A procedure for identifying the plastic behavior of single crystals from the local response of polycrystals. Acta Materialia, 51:5477-5488, 2003.

30. H. Lim, J. D. Carroll, C. C. Battaile, T. E. Buchheit, B. L. Boyce, and C. R. Weinberger. Grainscale experimental validation of crystal plasticity finite element simulations of tantalum oligocrystals. International Journal of Plasticity, 60:1-18, 2015.

31. F. Kroupa. Plastic deformation of BCC metals with special reference to slip geometry. Ecole d'été de Pont-à-Mousson, Nancy, 1967.

32. V. Vitek. Theory of the core structures of dislocations in body centred cubic (BCC) metals. Crystals lattice defects, 5:1-34, 1974. 
33. C. Hennessey, G. M. Castelluccio, and D. L. McDowell. Sensitivity of polycrystal plasticity to slip system kinematic hardening laws for Al 7075-T6. Materials Science and Engineering A, 687:241 248, 2017.

34. P. McCormick. Theory of flow localisation due to dynamic strain ageing. Acta Metallurgica, 36:30613067, 1998.

35. S. Zhang, P. McCormick, and Y. Estrin. The morphology of portevin-le chatelier bands: finite element simulations for Al-Mg-Si. Acta Materialia, 49:1087-1094, 2000.

36. S. Graff, S. Forest, J.L. Strudel, C. Prioul, P. Pilvin, and J.L. Bechade. Strain localization phenomena associated with static and dynamic strain ageing in notched specimens: experiments and finite element simulations. Material Science and Engineering A, 387-389:181-185, 2004.

37. M. Marchenko, M. Mazière, S. Forest, and J. L. Strudel. Crystal plasticity simulation of strain aging phenomena in alpha-titanium at room temperature. International Journal of Plasticity, 85:1-33, 2016

38. S. Ren, M. Mazière, S. Forest, T. F. Morgeneyer, and G. Rousselier. A constitutive model accounting for strain ageing effects on work-hardening. Application to a $\mathrm{C}-\mathrm{Mn}$ steel. Comptes Rendus Mécanique, 345:908-921, 2017.

39. Z-set package. Non-linear material \& structure analysis suite, www.zset-software.com. 2013.

40. H. Lim, J. D. Carroll, C. C. Battaile, B. L. Boyce, and C. R. Weinberger. Quantitative comparison between experimental measurements and CP-FEM predictions of plastic deformation in a tantalum oligocrystal. International Journal of Mechanical Sciences, 92:98-108, 2015.

41. A. Marais, M. Mazière, S. Forest, A. Parrot, and P. Le Delliou. Identification of a strain-aging model accounting for luders behaviour in a C-Mn steel. Philosophical magazine, 92:3589-3617, 2012.

42. S. Graff, S. Forest, J.L. Strudel, C. Prioul, P. Pilvin, and J.L. Bechade. Strain localization phenomena associated with static and dynamic strain ageing in notched specimens: experiments and finite element simulations. Material Science and Engineering A, 387-389:181-185, 2004.

43. H.D. Wang, C. Berdin, M. Mazière, S. Forest, C. Prioul, A. Parrot, and P. Le-Delliou. Portevin-le chatelier (PLC) instabilities and slant fracture in c-mn steel round tensile specimens. Scripta Materialia, 64:430-433, 2011.

44. L. Allais, M. Bornert, T. Bretheau, and D. Caldemaison. Experimental characterization of the local strain field in a heterogeneous elastoplastic material. Acta Metallurgica and Materialia, 42:38653880, 1994.

45. S. Suresh. Fatigue of Materials. Cambridge University Press, 1998.

46. A.F. Bower and K.L. Johnson. The influence of strain hardening on cumulative plastic deformation in rolling and sliding contact. Journal of the Mechanics and Physics of Solids, 37:471-493, 1989.

47. D.L. McDowell. Stress state dependence of cyclic ratchetting behavior of two rail steels. International Journal of Plasticity, 11:397-421, 1995.

48. M. Zhang, R.W. Neu, and D.L. McDowell. Microstructure-sensitive modelling: Application to fretting contacts. International Journal of Fatigue, 31:1397-1406, 2009.

49. M. Abdel-Karim and N. Ohno. Kinematic hardening model suitable for ratchetting with steady-state. International Journal of Plasticity, 16:225-240, 2000.

50. S. Bari and T. Hassan. Anatomy of coupled consitutive models for ratchetting simulation. International Journal of Plasticity, 16:381-409, 2000.

51. J.L. Chaboche and J. Lemaitre. Mécanique des matériaux solides. Éd. 3. Dunod, 2008.

52. S. Sinha and S. Ghosh. Modeling cyclic ratcheting based fatigue life of hsla steels using crystal plasticity fem simulations and experiments. International Journal of Fatigue, 28:1690 - 1704, 2006.

53. L. Priester. Grain Boundaries: From Theory to Engineering. Springer Series in Materials Science 172. Springer Netherlands, 1 edition, 2013.

54. J. Tong, L.G. Zhao, and B. Lin. Ratchetting strain as a driving force for fatigue crack growth. International Journal of Fatigue, 46:49 - 57, 2013.

55. D. Colas. Approche multi-échelles du vieillissement et du comportement mécanique cyclique dans le tantale. PhD, Mines ParisTech, 2013.

56. S Manchiraju, M Asai, and S Ghosh. A dual-time-scale finite element model for simulating cyclic deformation of polycrystalline alloys. The Journal of Strain Analysis for Engineering Design, 42:183200, 2007.

57. H. Lim, H. J. Bong, S. R. Chen, T. M. Rodgers, C. C. Battaile, and J. M. D. Lane. Developing anisotropic yield models of polycrystalline tantalum using crystal plasticity finite element simulations. Materials Science and Engineering: A, 730:50 - 56, 2018. 